\title{
Humanización de la calidad en la atención clínica en salud desde la perspectiva centrada en el paciente a partir de la Resolución 13437 de 1991
}

\author{
Humanization of clinical care quality in health from the patient perspective centered from \\ the Resolution 13437 of 1991
}

Julián Darío Santacruz-Bravo'1

1 Filósofo, Psicólogo, Esp. Gerencia y Auditoria de la Calidad en Salud. Docente Orientador I.E. José Antonio Llorente, Municipio de Cumbal, Colombia. e-mail: julisatb@gmail.com de la Resolución 13437 de 1991. Univ. Salud. 2016:18(2):373-384. DOI: http://dx.doi.org/10.22267/rus.161802.47

\begin{abstract}
Resumen
La humanización versus deshumanización de la calidad de la atención clínica en los servicios de salud, ha llamado la atención de todos los sectores de la sociedad colombiana en los últimos años. La falta de sensibilización frente a los procesos sociales, que incluyen un adecuado tratamiento de los procedimientos en salud, infiere la presencia de algunos elementos que atentan contra la calidad de vida de los usuarios. Este artículo busca interpretar hasta qué punto los derechos de los enfermos, tal como están concebidos, responden fielmente con la recuperación de la salud, y con ella, la humanización del servicio. Los primeros hallazgos de esta investigación demuestran cada vez más la insuficiencia de un modelo de calidad en la atención enfocado en el persona como proceso, por otro lado, el usuario de la salud sigue siendo entendido como un organismo enfermo, aislado, un cuerpo desprovisto de su singularidad y restringido al conocimiento técnico-científico que rodea la experiencia clínica lo que lo aleja cada vez mas de sus derechos fundamentales en el marco de una democratización de la salud. En conclusión, hablar de humanización en la calidad de la atención clínica en salud, es poner toda la producción de sus servicios al alcance de la mano de los pacientes, y no al contrario, recuperar la dignidad humana, no es otra cosa que, concentrar todos los esfuerzos en el sujeto del paciente y no en su exterior.
\end{abstract}

Palabras clave: Humanización de la atención; derechos del paciente; gestión de la calidad. (Fuente: DeCS, Bireme).

\begin{abstract}
The humanization versus dehumanization of the clinical care quality in the health services has attracted the attention of all sectors of Colombian society in recent years. The lack of awareness of social processes, which include a suitable treatment procedures in health infers the presence of some elements that threaten the quality of life of the users. This article seeks to interpret to what extent the rights of ill people, as they are designed, respond faithfully with the recovery of health, and with it, the humanization of the service. The first findings of this research show increasingly the inadequacy of a model of quality of care focused on the person as a process; on the other hand, the user of health is still being understood as an ill organism, a body devoid of its uniqueness, isolated and restricted to the technical and scientific knowledge that surrounds clinical experience, which every time takes the patient away from his or her fundamental rights in the framework of a democratization of health. In conclusion, to talk about humanizing the clinical care quality in health is to put the entire production of its services to the fingertips of the patients, and not the
\end{abstract}


opposite; restoring human dignity is nothing more than concentrating all efforts on the subject and not on the outside of the patient.

Keywords: Humanization of assistance; patient rights; quality management. (Source: DeCS, Bireme).

\section{El nacimiento de la clínica: "el enfermo como sujeto de su enfermedad vs el sujeto como accidente de su enfermedad"}

En un país como Colombia, resultaría imposible hablar del cumplimiento de los derechos de los enfermos, si estos no están precedidos y acogidos por el concepto de calidad, entendida como la satisfacción de necesidades y expectativas de los usuarios, pacientes o clientes proveyendo el mayor beneficio con un mínimo riesgo, dados unos recursos.

Ya desde los tiempos del juramento hipocrático, se planteaban aspectos relacionados con la importancia de conservar el concepto de humanidad; tal es el caso de principios como: el respeto al paciente, la confidencialidad, la accesibilidad y la competencia profesional. Con el código Hammuirabbi en 1683, se instauraban las primeras sanciones a las que se harían acreedores los galenos por aquellas prácticas que produjeran lesión, destrucción de un órgano o la muerte. En este mismo sentido, hablar de humanización en la atención clínica en salud, bajo el marco de los derechos del paciente, implicaría analizar más de cerca el concepto mismo de humanismo. Según Hamburger F. Álvaro, fue "F.I. Niethammer, quien lo utilizó por primera vez para indicar el área cultural a la que se dedican los estudios clásicos y el espíritu que les es propio, en contraposición con el área cultural que cubren las disciplinas científicas"1.

De esta manera, autores como Reale y Antiseri, sostienen que: "humanista es quien se dedica a enseñar y cultivar la gramática, la retórica, la poesía, la historia y la ética (...) concordando en este sentido con Cicerón y Gelio, pensadores latinos para quienes humanitas, significa aproximadamente lo que los griegos habían expresado con el término paideia, es decir, educación y formación del hombre"1.
Igualmente, el nacimiento de la clínica se convertiría para efectos de una atención de la salud, en un referente crucial para el ejercicio de la medicina, toda vez que su desarrollo nos exige una mirada más profunda a través de la historia, más, si está en juego la manera cómo los médicos de la época trataban a los enfermos, así como su influencia en los tiempos modernos.

Mucho antes de terminar el siglo XVIII existía la clínica. Se cree que la medicina encontró en ella su origen. La relación entre el sufrimiento y la cura ha existido desde el nacimiento de la humanidad. Como bien lo anota Foucault: "esta relación era de instinto y sensibilidad, más aún que de experiencia; estaba establecido por el individuo, por él mismo y para sí mismo, antes de entrar en una red social (...)"2, sin embargo, esta relación no estaba mediada por el saber, lo que hace que no tome conciencia de su accionar, hasta que es transmitida de generación en generación como una forma de conocimiento, en el cual cada individuo es a la vez el sujeto y el objeto de conocimiento, así todo el mundo practicaba esta medicina, hasta que según Foucault ${ }^{2}$, este saber entró en decadencia cuando paso a ser parte de un grupo privilegiado, produciendo una disociación absoluta entre lo que él llamo "mirada y palabra", lo que se había sabido, no se comunicaba ya a los demás, a no ser que pase por el esoterismo de la época".

En aquellos tiempos, el arte de la medicina se enseñaba en presencia de su objeto y los jóvenes aprendían la ciencia médica en el lecho del enfermo, estos, narra la historia: "estaban alojados con mucha frecuencia en el domicilio mismo del médico y los alumnos acompañaban a los maestros, mañana y tarde a la visita de sus clientes"3.

Curiosamente en el siglo XVIII, la clínica, no estaba abierta a todo lo que venga, como puede estarlo la práctica cotidiana de un médico, ni tampoco 
especializada, como estará en el siglo XIX: "no es ni el dominio de lo que se ha escogido para estudiar, ni el campo estadístico abierto de lo que se está consagrado a recibir; se vuelve a cerrar sobre la totalidad didáctica de una experiencia ideal (...) No tiene la obligación de mostrar los casos, sus puntos dramáticos, sus acentos individuales, sino de manifestar en su recorrido complejo, el círculo de las enfermedades"2.

Un ejemplo de ello, es la famosa clínica de Edimburgo (1720), la cual estaba constituida de tal modo que se encontraban allí reunidos los casos que parecen más adecuados para instruir; Michel Foucault pensaba que: "antes de que se produjera el encuentro entre el médico y el enfermo, de una verdad por descifrar y de una ignorancia, para poder ser, la clínica debe formar constitucionalmente, un campo nosológico enteramente estructurado...el papel del médico en el hospital es descubrir la enfermedad en el enfermo...en la clínica-agrega el sabio-, se tratan las enfermedades a la inversa cuyo portador es indiferente: lo que está presente es la enfermedad misma, en el cuerpo que le es propio y que no es el del enfermo, sino el de su verdad... el enfermo es solo aquello a través de lo cual se da el texto a leer, a veces complicado y enredado(...) En el hospital, el enfermo es sujeto de su enfermedad, es decir que se trata de un caso; en la clínica, en la cual no se trata sino del ejemplo, el enfermo es el accidente de su enfermedad, el objeto transitorio del cual ésta se ha apropiado"2.

¿Acaso estos indicios dan cuenta de las primeras manifestaciones de lo que más tarde se denominaría como la "humanización de la salud"? Por primera vez los médicos de la época entre ellos Tissot ${ }^{4}$, deciden confiar cada enfermo de la clínica a los estudiantes, "son ellos y solo ellos quienes lo examinarán con honestidad, con dulzura, con esa bondad que es tan confortante parta esos pobres infortunados".

Sin embargo, no todo termina con la valoración física del enfermo, así lo hace notar Foucault en sus reflexiones, cuando hace hincapié en que la clínica "no hace del enfermo el inventario de un organismo afectado, es decir, la mirada que recorre un cuerpo que sufre, no alcanza la verdad que busca, sino pasando por el momento dogmático del nombre, en el cual se recoge una doble verdad: esta oculta, pero ya presente en la enfermedad, está cerrada, pero claramente deducible de la conclusión y de los medios..." ${ }^{2}$.

En el siglo XVIII, no hay clínica que sea pedagógica y ésta, incluso, se practicaba de forma restringida; la clínica, no toca sino a esta instrucción, en forma limitada, que es dada por el maestro a sus alumnos; no es una experiencia en sí misma, sino un legado para las futuras generaciones. Pero, cuidado, estas lecciones dadas por el maestro, también podían volverse contra él; la concepción de la época creía que si un profesor se equivocaba, sus errores son revelados inmediatamente por la naturaleza cuyo lenguaje es imposible apagar o alterar; a menudo incluso, estos errores, son más útiles que sus éxitos y hacen más ineficaces las imágenes que, sin esto, quizá no hubieran sido en ellos, sino impresiones pasajeras. Así Foucault, vaticina cambios bruscos parta la clínica del futuro: “(...) desprendida del contexto histórico en el cual había nacido, ya no va a tener un campo de acción limitado en el cual se dice un saber, sino coextensivo con aquel en el cual nace, se prueba y se realiza: formará un cuerpo con el todo de la experiencia médica. Se armara de nuevos poderes, desligada del lenguaje a partir del cual se le profería como lección, liberada por un movimiento de descubrimiento (...)"2.

\section{Del concepto de humanidad y los derechos de los enfermos}

En los últimos años del siglo XVIII, afirma Etchegoyen ${ }^{5}$, cuando Pinel (1745 -1826) implanta su heroica reforma hospitalaria, introduce un enfoque humano, digno y racional, de gran valor terapéutico en el trato con el enfermo. Posteriormente, su brillante discípulo Esquirol (1772 - 1840), crea el llamado tratamiento moral, consistente en un conjunto de medidas "no físicas", que preservan y levantan la moral del enfermo, especialmente el hospitalizado, evitando los graves artefactos iatrógenos del medio institucional. 
Más adelante, aparecen grandes defensores de la calidad en salud como Florence Nightingale (1850), Ernest Codman (1914), Donabedian (1966), Lee y Jones (1990) o la aparición de la Joint Comisión on Acreditation of Health Care Organizations, entre otros, quienes no solo se preocuparon por analizar los niveles de morbimortalidad de los pacientes, teniendo en cuenta el ejercicio profesional, así como la ética por la calidad de su trabajo y la buena atención clínica, sino también por la importancia de incluir en ellos el componente de dignidad $y$ humanización en el enfermo, si por humanización se entiende como una acción ética que tiene que ver con los valores que conducen nuestra conducta en el ámbito de la salud, y estos valores nos llevan a diseñar políticas, programas, realizar cuidados y proteger las relaciones en sintonía con la dignidad de todo ser humano.

Con la adopción formal por parte de las Naciones Unidas sobre la Declaración Universal de los Derechos Humanos ${ }^{6}$ el 10 de diciembre de 1948, se buscó concientizar al mundo entero sobre la importancia de los derechos básicos para los habitantes del planeta. Los derechos humanos abarcan tanto los derechos civiles, como políticos de los individuos, los derechos sociales y económicos, pero también los que en este momento acaparan nuestro interés, los de la atención en la salud (Arts. 22 al 27). Aquí, los profesionales de la salud prometen mantener los más altos estándares de integridad personal y competencia, tener compasión por las personas que requieren de su atención, este conocimiento que les caracteriza, que busca convertirlos en profesionales de la salud con poderes y responsabilidades especiales, los ha llevado a convertirse en un sector muy importante en lo que tiene que ver con la atención a la comunidad.

Desde el artículo primero de los derechos humanos, donde se enuncia que cada ser humano: "nace libre e igual en dignidad y derechos"6, el documento enumera las libertades críticas que llenan el espacio que rodea a cada hombre, mujer y niño sobre la tierra. ¿Qué se busca en esos compromisos y qué ofrece la declaración de los derechos de los enfermos?, ¿acaso contempla el reconocimiento de una relación terapéutica capaz de sostener, desde el deseo, no tanto un cuerpo enfermo (en tanto cuerpo de necesidad), sino un sujeto fracturado (en tanto sujeto en falta)?, ¿existe la preparación académica, profesional, pero ante todo humana, para comenzar a pensar por primera vez en un modelo de atención amparado en una democratización de la salud?

\section{Colombia y los derechos de los pacientes}

El ejercicio de la salud en Colombia, a pesar de que hay pocos registros en materia de humanización de la salud, se ha convertido para efectos de la calidad de la atención clínica, en el ejemplo más claro de lo que últimamente los usuarios reclaman de las instituciones: un tratamiento más humano y digno. Esa concepción tradicional que aún manejan las facultades de medicina donde "el doctor sabe más", "el doctor decidirá", o el "paciente no necesita saber", siguen siendo temas controvertidos en el entrenamiento del profesional y juegan un papel legítimo en la práctica médica, en instancias y momentos particulares. Las discusiones sobre ética médica, hacen énfasis en la noción de autonomía y derechos del paciente, vista como un actor independiente, que merece respeto $y$ responsabilidad profesional, simplemente porque es un ser humano. Dentro del marco de los derechos humanos, esta noción se representa con las palabras "dignidad humana", o el atributo de los seres con derechos ya sea que sepa que los tiene o no'.

La acreditación en salud en Colombia, según su director, el Dr. Carlos E. Rodríguez ${ }^{7}$, viene proponiendo un acercamiento al tema; se trata de proponer soluciones prácticas y visibles a los problemas reconocidos e impulsar a las instituciones en el abordaje de la problemática.

Este aporte inicial, es por decirlo menos reduccionista, pues parte de mirar el problema dentro de las instituciones de salud, se concentra en un tipo de instituciones (los prestadores). Aunque sería esencial tener un abordaje similar para las aseguradoras y para entes territoriales, aborda superficialmente el problema estructural del talento humano para la salud y no da pistas 
sobre soluciones; se limita a proponer estándares que nos ayuden en cuanto metas concretas. Se pretende con ello estimular la discusión y generar la necesidad de actuar.

La propuesta es sencilla, afirma el experto: “(...) proponemos la construcción de una política explícita de humanización de la atención que aborde los problemas señalados aquí y otros omitidos por ignorancia o limitaciones de espacio. Se reconocen en este texto, al menos tres vertientes del abordaje de la humanización en los servicios de salud. Una primera vertiente es el trabajo que desde hace décadas realizan algunas comunidades religiosas, por ejemplo los hermanos Camilos, los hermanos de San Rafael, las hermanas de la Presentación, entre otras comunidades católicas, que han dedicado buena parte de sus esfuerzos al cuidado de la salud y especialmente a aspectos de la humanización del servicio tales, como la acogida (hospitalidad), el diálogo, el apoyo espiritual y emocional, la escucha activa, el consuelo ante la tragedia que suele ser la enfermedad, entre otros aspectos para resaltar (...) Una segunda vertiente es la inspirada en la reivindicación de los derechos y los deberes, la autonomía y el respeto de la voluntad de los pacientes, como eje de la atención humanizada.

Este segundo abordaje, parte especialmente de los principios y aplicación de la bioética y de la reflexión jurídica y filosófica (...) La tercera vertiente es una tendencia intermedia que ve la humanización de la atención en salud, como un reto de calidad de las instituciones, no solo en la humanización de la atención al usuario sino también en el abordaje de la problemática de los trabajadores de la salud. La acreditación en salud se inscribe en esta tercera vía, en la que durante años hemos ido incorporando aspectos de las dos vertientes anteriores, generalizando su contenido para que en un caso sea una visión más incluyente ,y en el otro, menos abstracta y jurídica. La humanización surge entonces como una política institucional que debe dar respuesta a necesidades singulares de usuarios $y$ trabajadores $(\ldots)^{\prime \prime}$.

Pero, ¿realmente importa para los profesionales de la salud, así como las instituciones para las que trabajan, aprender a ver en el paciente como un otro, como un ser autónomo, para el que cada día tienen que esforzarse en aprender de él, escucharlo y entenderlo? Muchas líneas de investigación y evidencia sugieren que así sea; parece haber, sin embargo, una conducta dirigida a centrar la atención más en la enfermedad que en el cuidado de la salud, desatendiendo a la persona en su integridad. Las percepciones, sentimientos y emociones ya no son tenidas en cuenta, el uso reparador de la palabra comunicativa y comunicante se perdió completamente en el horizonte lingüístico de la clínica y la ética para dar paso a otro tipo de significantes instaurados en el borde mismo de un inconsciente mutilado y sin representación simbólica: el desconocimiento absoluto de "ese otro" y de sus derechos, el sujeto borrado, el sujeto anulado.

T. S. Eliot ${ }^{8}$, habla de la necesidad de ver en cada uno de los otros, como a "un extraño", un desconocido acerca del cual no se puede asumir nada, ni presumir conocimiento previo, es lo que Martin Buber resumiría de una manera estética al describir la única parte inseparable de una persona por definición: “(...) cuando vamos por nuestro camino y encontramos a un hombre que viene hacia nosotros, por su camino, conocemos nuestro camino solamente y no el suyo; para el suyo viene la vida, para nosotros solo el encuentro $(\ldots)^{\prime \prime}$.

\section{Hacia un programa de humanización de la salud pensado en los derechos de los pacientes: estructura, proceso y resultado}

El paciente debe ser escuchado e introyectado con todos los canales de comunicación que vayan más allá de las consabidas "encuestas de satisfacción", "buzones de sugerencias" o de "quejas y reclamos", etc., estos solo recuerdan el regreso a un sistema oscuro y positivista, donde todos los involucrados conocen el principio, pero nunca el final, precisamente porque, este tipo de sistemas, están diseñados de tal manera que haya un solo espectador que regula el ordenamiento total de la empresa, tal y como lo describiría muy bien en su momento Foucault a propósito del "Panóptico" de Bentham: “(...) Basta entonces 
situar un vigilante en la torre central y encerrar en cada celda a un loco, un enfermo, un condenado, un obrero o un escolar (...) La complejidad de la práctica clínica actual contiene muchas posibilidades diagnósticas y terapéuticas, en el curso de las cuales un profesional de la salud está obligado a comunicar cercana y enfáticamente de acuerdo con las necesidades de los pacientes o encontrará desconfianza, y potencialmente, pobres resultados en su profesión. Llevar una historia adecuada, dejar solo o acompañar a un paciente requiere de una esforzada y educada capacidad para relacionarse salvando barreras significativas" 10 .

Por eso, se habla de una estructura que incluye la creación de lo que se llama un Centro de Escucha para la Humanización de la Atención al Paciente, el cual está coordinado por un tutor (no "jefe") de área con funciones estrictamente humanitarias y de calidad de la atención clínica en salud, y cuya cobertura, estará dirigida a dos tipos de población: los sujetos (no "clientes") y los promotores (no "prestadores") de los servicios de salud, de modo que la asistencia dentro, y aún fuera del consultorio, adquiera un sentido "holístico" para los que aspiran a satisfacer sus expectativas en la atención.

El Centro, además, tiene la función de difundir una cultura de la salud a través de la publicación de artículos científicos, cuya política este dirigida a rescatar a la salud y con ella, al paciente, como un ser humano integral, más allá de la enfermedad o trauma que lo aqueje, con el principio de que: no hay paciente desmotivado, insatisfecho, sino "mal informado", "desconocido", lo que significa que ahora la calidad en la atención clínica ya no va a depender solamente, de qué tan satisfechas sean las necesidades del usuario, sino también de qué tan bien este educado dentro de la cultura de la salud.

La posición de la medicina en una sociedad como la colombiana, aún está en desarrollo, ya que las barreras socioeconómicas para el acceso y calidad de la salud, han apurado a la población general a hacer ajustes críticos en materia de políticas sanitarias. El personal de salud está ahora impulsado a reforzar sus habilidades como comunicadores, defensores de los pacientes, de sus derechos y conciliadores entre los sistemas y sus expectativas. Es un deber del personal de salud recolectar esta información, protegerla, considerarla y resolverla para tener un conocimiento más cercano a esas necesidades y expectativas de los pacientes, mejorar la gestión de los servicios de salud con un verdadero enfoque hacia el usuario.

En este orden de ideas, el proceso en cada evento de salud, desde la sesión clínica, hasta procesos de mayor complejidad, deberán ir acompañados de una ficha conceptual donde cada profesional de la salud, de acuerdo a la especialidad, diseñará e integrara como mínimo los siguientes aspectos: motivo y motivación de consulta, asistencia y calidad del servicio. La primera, cuya alianza terapéutica deberá adoptar el siguiente decálogo: los pacientes son personas, cuyos derechos incluyen: respetar su singularidad, evaluar con objetividad, rescatar el análisis interpersonal y no la crítica, convertir el trabajo en una experiencia de conocimiento mutuo, compartir los triunfos con nuestros pacientes, aprender a escucharlos, fomentar el cambio estimulando la creatividad (no la "experimentación") y el aprendizaje, cultivar la prudencia más que la astucia, a veces es mejor sacrificar la perfección a cambio de la eficacia pero con enfoque humano y diferencial, es necesario promocionar las ventajas materiales y sociales que vayan en beneficio de la salud relacionándolas estrechamente con la contribución a los resultados, invertir en la formación de nuestros pacientes, aprendiendo a no dejar de aprender de ellos, el trato respetuoso hacia el paciente implica tratarlo como a un igual.

Se trata de una asistencia cuya impresión está influenciada por los valores, las experiencias previas y el concepto del papel que los pacientes tienen sobre los sistemas de salud, de sus proveedores, de los riesgos y beneficios de la atención recibida, la cual debe establecer un concepto claro de la calidad que debe concebirse para la atención que se quiere brindar, es decir empezar a construir un nuevo concepto de liderazgo "integral", con profundo sentido social y co-participativo (no de "empresa de negocios"), de una verdadera y sostenida atención al usuario, 
de atención clínica, de tratamiento de la enfermedad, como un lugar abierto y de permanente comunicación que nos hace desarrollarnos personal y profesionalmente. Una visión de que los servicios y productos de calidad en salud están al servicio del paciente, y no al contrario; para ello, es necesario un trabajo de formación, de implicación, de responsabilidad y pertinencia, y cuando se trata de interactuar con personas, la asertividad y la creatividad serán cualidades que los profesionales que las poseen, hagan que los procesos se reinventen permanentemente y avancen.
Es imprescindible incluir un concepto de calidad, que garantice un servicio antes, durante e incluso después del procedimiento; vale la pena destacar dos aspectos interesantes que plantea M. Herrera Carranza, cuando afirma que para impulsar la calidad se hace necesario: "comprender que estamos inmersos en una época de cambio de cultura sanitaria, caracterizada por la aparición de nuevas tendencias en las actitudes de los usuarios, de los profesionales y de las instituciones. El hospital con todos sus servicios debe adaptarse a estas innovaciones si quiere avanzar (...)"11; aquí algunos ejemplos:

Cuadro 1. Tendencias sanitarias actuales

\begin{tabular}{ll}
\hline \multicolumn{1}{c}{ Presente } & \multicolumn{1}{c}{ Futuro } \\
\hline Necesidades profesionales & Necesidades de la población \\
Curar enfermedades & Promoción de la salud \\
Frialdad clínica & Ambiente cálido \\
Hospital con todos sus servicios & Centros de responsabilidad \\
Servicios aislados, escasa coordinación & Unidades funcionales, centros operativos \\
Actividades diagnósticas y terapéuticas & Mejora continua de la calidad \\
Paciente interno & Paciente externo \\
Paciente cautivo & Paciente cómodo \\
Público poco informado & Consumidor exigente \\
Indiferencia ante el anciano & Sensibilidad ante el anciano \\
'No se puede pasar....' & 'Pase, no se va a perder..... \\
\hline
\end{tabular}

Fuente: Herrera Carranza, M. ${ }^{11}$

El segundo componente puntualiza Herrera Carranza tiene que ver con: "transmitir la idea de provecho clínico, de contribución a la mejora de resultados y no de carga burocrática impuesta por la "administración". A la palabra control hay que quitarle el cariz represivo o sancionador y darle un significado de vigilancia de la actividad, de dominio de la situación por corregir y progresar"11.

$\mathrm{Al}$ incorporar las perspectivas de los pacientes y los proveedores o profesionales en las iniciativas de mejorar la calidad de la atención clínica, las autoridades que diseñen las políticas y de los directores del programa lograrán tener un mejor conocimiento de las necesidades y dificultades de ambos grupos de tal manera que si se comparte el objetivo de mejorar la calidad de la atención, se conseguirá finalmente el aumento en el uso de los servicios, con capacidad de sostenimiento, optimización de la salud tanto de quienes reciben la atención como de quienes la brindan, tal y como lo establecía Donabedian, a propósito de garantizar un buen resultado en la atención en salud a partir de un concepto de calidad :

"la calidad de la atención debe tener las características de integralidad y sistematicidad para que sea confiable y válida, considerando componentes adecuados a la naturaleza del servicio tanto en la estructura como del proceso y diría que con más énfasis- en el resultado" 12 .

Todo profesional de la salud que gasta parte de su vida trabajando con grupos humanos desfavorecidos, no puede escapar a las limitaciones impuestas por la contradicción que actualmente existe entre la salud que se presta y los derechos del paciente que se promulgan. Aunque se viola cada día, en cada país del mundo estos derechos, el artículo 25 de los derechos humanos declara: "cada uno tiene derecho a un estándar de vida adecuado para la salud y el bienestar de sí mismo y de su familia..."6. ¿Así 
pues, dónde comienzan los derechos de los enfermos?, quizá en aquellos nichos particularmente insospechados, en pequeños lugares cerca de casa, la escuela o el centro de salud, tan próximos, y al mismo tiempo, tan lejanos que no pueden ser vistos en el contexto del mundo.

Aún existe el universo de la persona individual: el barrio en el que vive, la escuela a la que asiste, el lugar donde trabaja, y es aquí, donde finalmente descansa lo que puede ser el eje central del profesional de salud, la esencia misma que se cree puede aproximarnos a dar un sentido a la resignificación del modelo de la calidad de la atención, un modelo, cuya posición defenderá la tesis, según la cual, no hay mejor lugar para empezar a impartir una conciencia de la dignidad humana, que en el pequeño universo de la relación médico-paciente, ahí donde la razón de ser, es la misma persona y no su exterior.

Entonces, ¿qué son finalmente, los derechos del paciente?, ¿cuál es la relación entre el derecho a acceder a un servicio de salud y una buena atención clínica? Intentar definir la naturaleza de estos derechos, y su relación con la atención clínica en salud, es una tarea dispendiosa. Incluso si se habla de los mismos derechos humanos, el trabajo no es menos difícil. Nino afirmaba que: "a pesar de los innumerables intentos de análisis, el lenguaje de los derechos sigue siendo muy ambiguo, poco riguroso y muchas veces es usado de manera retórica. Se hablan de derechos naturales, de libertades básicas, de derechos básicos, derechos fundamentales, etc."13.

Después de hacer un recorrido histórico por el tema, Pérez Luño afirma que: "la ciencia de los derechos del ser humano, se refiere a la persona, en especial al trabajador, que vive en un Estado, y que, acusado de una infracción o víctima de guerra, se beneficia con la protección de la ley, gracias a la intervención de un juez nacional o de las organizaciones internacionales, $\mathrm{y}$ cuyos derechos, especialmente el derecho a la igualdad, se armonizan con las exigencias del orden público"14.
Es más, la Resolución 13437 de 1991, del Ministerio de Salud en Colombia, declara que: "todo paciente debe ejercer sin restricciones de ninguna clase su derecho a la salud"15, sin embargo, resulta paradójico pensar, como a pesar de que la salud queda consagrada como un "bien fundamental", no lo es, en cambio, como un "derecho fundamental", a menos que, mediante una acción de tutela, se llame la atención sobre algún hecho que atente, contra la vida misma.

Entonces, se convierte en un derecho fundamental, si y solo si, su estado se ve gravemente amenazado. ¿Qué hacer frente a esta ambigüedad y variedad de sentidos, mientras los sujetos, aparte de ser ignorados en su atención, todavía siguen esperando, e incluso, muriendo a las afueras, o aún, dentro de los hospitales? Si el paciente tiene derecho a: "recibir un trato digno, respetando sus creencias y costumbres, así como las opiniones personales que tenga sobre la enfermedad que sufre..." ${ }^{15}$, ¿por qué cada día es más difícil acceder a los servicios de salud?, ¿gozan los derechos de los enfermos de los principios de eficiencia, universalidad y solidaridad?

La Defensoría del Pueblo advierte en un informe preliminar sobre el incremento sostenido en el número de tutelas en salud, que para 2014 alcanzo un total de 118.281, que representa el $23,7 \%$ del total de este tipo de acciones jurídicas interpuestas por los colombianos. Según los datos que la entidad viene consolidando, preocupa que entre 2010, cuando se presentaron 94.502 acciones de tutela en salud, y 2014, el número de acciones para reclamar por este derecho fundamental aumento un 25,2\%. El panorama llama la atención en el marco de la conmemoración del Día Mundial de la Salud, dado que el $70 \%$ de las solicitudes de los ciudadanos en materia de violaciones a su derecho fundamental está relacionado con solicitudes incluidas en el Plan Obligatorio de Salud (POS) ${ }^{16}$.

¿Acaso llegó la hora de redimensionar los derechos de los pacientes sobre la base de establecer una política en salud donde el equilibrio en recursos humanos y técnicos se incline hacia los primeros, diseñando $\mathrm{y}$ 
potenciando todos los valores que dignifiquen la condición humana? Si esto es así, se hace necesario, pues, la instauración de una política de los derechos cuyos valores deben llamar la atención de todas las instituciones prestadoras de servicios de salud donde la persona es el centro alrededor del cual girarán todos los procesos, estructuras y acciones, tanto de la organización como del personal sanitario y asistencial, una política que imparta una educación sanitariopreventiva y previsiva del paciente que debe ser tan importante como la propia asistencia, así como el grado de confianza que recibe el paciente del tratamiento terapéutico respectivo.

Pero, esta tarea no solo es de quienes ofrecen un servicio, es también una responsabilidad del quien la recibe, que convertido en un agente político, tiene dos tareas, por un lado, es un beneficiario permanente de sus derechos, y por otro, es un asiduo defensor y multiplicador de los mismos, esta vez con un deber: tener un conocimiento más participativo y amplio de su proceso de cambio antes, durante y después de la enfermedad, en que la transmisión e intercambio de aprendizajes, tanto en conocimientos y habilidades, actitudes y emociones sean de vital importancia para el trabajo en equipo, tanto dentro como fuera de la relación prestadorpaciente.

La salud, en este orden de ideas, es el resultado de los cuidados propios y hacía los demás, de la capacidad de tomar decisiones y controlar la vida propia y de asegurar que la sociedad en la que se convive goce de una buena calidad de vida, una sociedad capacitada y consciente de la importancia de desarrollar, junto a los profesionales de la salud, sus máximas potencialidades, la adopción de hábitos y estilos de vida saludables, fomentando su cultivo y seguimiento, fortaleciendo permanentemente las actitudes y aptitudes tanto individuales como colectivas, comunitarias e institucionales para reducir la desigualdad y promover el desarrollo de la salud plena.

El trato interpersonal entre el promotor de la salud y su paciente, será algún día, un verdadero asunto de salud pública, precisamente porque los profesionales de la salud cada día deben tener actualizados algo más que sus conocimientos científicos, algo que vaya más allá del fármaco, la radiografía o la prueba de laboratorio, se trata nada más y nada menos que de la subjetividad, que comprende el continente humano, un horizonte todavía oculto a la razón instrumental de la "frialdad clínica", una poética del desenmascaramiento que amenaza levantar el velo de aquello a lo que más infunde miedo y resistencia: redescubrirnos en la mirada del otro, en su asombro, por demás perdido, que todavía debe producirnos la atención a un ser humano.

Si el paciente tiene derecho a: “(...) que se le preste durante todo el proceso de la enfermedad, la mejor asistencia médica disponible, pero respetando los deseos del paciente en caso de enfermedad irreversible"15. Entonces, ¿por qué hoy en día es cada vez más difícil adelantar el llamado rapport ("calidez y confianza") en el encuentro con el paciente?, o en el peor de los casos, ¿por qué un porcentaje considerable de pacientes, además de no recibir una buena atención, son mal diagnosticados, y por consiguiente, acompañados de un tratamiento equivocado? La carta de los derechos de los pacientes, el código de ética y el código de conducta para el personal de salud, entre otros, son elementos esenciales para el desarrollo de la humanización de la atención, sin embargo, antes deben ser bien comprendidos y asumidos por los directivos y por los trabajadores de las dependencias de las unidades de salud.

Entre las tres grandes categorías de derechos que la historia recrea, el profesional de la salud a veces olvida que, al igual que en los derechos civiles, sociales o económicos, se encuentra, antes que todo, con el derecho a la vida, pero con un ingrediente esencial que, hoy muchas empresas, instituciones o profesionales de la salud, nunca se detienen en reflexionar: no se trata solo de cuidar la integridad física frente a las amenazas externas, sino de garantizar de manera oportuna los medios para poder vivir de manera digna. Según la lógica de la tradición liberal, afirmar que el individuo tiene derecho a vivir, significa que el poder público debe poner a su disposición todos los medios para neutralizar las acciones violentas 
que pongan en riesgo su subsistencia; sin embargo, la posibilidad real de vivir depende finalmente, de sus destrezas y habilidades para encontrar en el mercado los medios de subsistencia.

En palabras de Angelo Papacchini: "con los derechos sociales ya no se trata de definir un ámbito de lo que él llama 'libertad negativa' en contra de un poder político siempre más fuerte, organizado y amenazante, para él, la tarea principal es la de exigir la intervención de este poder para que el individuo pueda realizar sus potencialidades en un marco de oportunidades para dos"17.

Papacchini17, habla del paso de una "libertad negativa" a una "libertad positiva", entendidas ambas como la posibilidad de pasar de una visión pesimista del poder a una concepción que no excluye su posible uso para redistribuir las riquezas y para realizar programas de bienestar social.

¿Acaso las necesidades insatisfechas de muchos, no sugiere la apertura de unos derechos, en relación con aquellos que poseen más de lo que necesitan?, ¿acaso la noción de lo que se conoce como "Estado social de derecho", no avala la razón de ser de los derechos sociales que se justifica y legitima en la obligación de asegurar las demandas de bienestar de los ciudadanos, incluyendo la atención clínica? Desafortunadamente, parece ser que en la medida en que nos resulte difícil delimitar el concepto de "atención y satisfacción de necesidades", será aún más exhaustivo definir el alcance de estos derechos, es decir, definir las necesidades mínimas de salud de la población es una tarea técnicamente posible, lo verdaderamente complejo, es definir lo que significa en cada caso el derecho a los medios indispensables para lo que se denomina una atención clínica en salud.

Los profesionales de la salud, y las mal llamadas empresas para las que trabajan, tendrían, entonces, la encomiable labor de dedicar todos los esfuerzos posibles para adelantar esta tarea, de manera estratégica, inteligente y más humana, de acuerdo a la dinámica de las necesidades y en la disponibilidad de recursos. Como bien lo anota Papacchini: "los derechos civiles son de realización inmediata, los derechos sociales, en cambio, son pensados como demandas que se irán realizando de manera progresiva, de acuerdo con la disponibilidad de recursos hasta lograr la plena efectividad (...) se parece a una idea regulativa en sentido Kantiano, son un deber ser, más que un ser..."17.

¿Cómo instaurar, finalmente, un modelo de calidad de la atención clínica en un servicio de salud, visto bajo el crisol de la humanización?, ¿existen serias tendencias y movimientos mundiales intelectuales, a largo plazo, centrados en el rescate de la persona humana, en la recuperación de su dignidad, y en devolverle a esta, su valor intrínseco? Interpretando a Hamburger ${ }^{1}$, se estaría asistiendo a lo que él llama: "la era de la revolución humana", en la medida en que hace de la persona y de su realidad, el punto central de sus estudios, preocupaciones y esfuerzos, por eso es preciso despertar una conciencia humanista en el ámbito de la atención clínica en salud, haciéndose necesario darles de nuevo el carácter humanizante a los derechos del paciente, siendo, por ejemplo, la ética una herramienta crucial para lograrlo.

Afirmar que ya no hay pacientes al servicio de la producción de los servicios de salud, sino que la producción de los servicios de salud, están al servicio de los pacientes, significa ponerlos a ellos en el centro mismo del proceso de la calidad humana para devolverle su dignidad, y no como en nuestro caso, que cobijados bajo la Ley 100 de 1993, y aún en la 1122 de 2007, el ciudadano promedio, pasa de convertirse de un capital subvalorado del sistema de aseguramiento en salud, ya que este depende de aquél, a un usuario cuya calidad de la atención sigue siendo de un nivel muy discreto; quizá, Colombia haya ganado en cobertura pero definitivamente ha cedido terreno en calidad, dando como resultado el desmejoramiento de las condiciones de vida y trabajo del recurso humano asistencial, así como la insatisfacción total de la atención que reciben los pacientes. 
Con base en los conceptos de humanización que se han analizado, la tarea, entonces, del profesional de la salud es "educar y formar", es decir, hacer que él sea aquello que debe ser de acuerdo con su naturaleza específica. Desde esta óptica, humanización y salud serían términos íntimamente ligados, lo que hace recordar una expresión relevante que ya se convierte en un principio filosófico de la salud y que hará parte, junto a la ética, en el manual de procedimientos a seguir a la hora de mejorar la calidad de la atención clínica en salud, hablamos de la llamada salud holistica ${ }^{18}$ que se refiere precisamente a la salud global, integral o total del ser humano. La holística, del griego holos, que significa "todo o totalidad", es un adjetivo que se refiere a la manera de ver las cosas enteras, en su totalidad, en su conjunto, en su complejidad.

En conclusión, es posible recuperar la dignidad humana a partir de concentrar todos nuestros esfuerzos en la persona del paciente y no en su exterior; si todo paciente le asiste el derecho a: “(...) disfrutar de una comunicación plena y clara con el médico, apropiada a sus condiciones psicológicas y culturales, que le permitan obtener toda la información necesaria respecto a la enfermedad que padece, así como a los procedimientos y tratamientos que le vayan a practicar y el pronóstico y riesgo que dicho tratamiento conlleve $(. . .)^{18}$, entonces, hablar de humanización en la calidad de la atención clínica, no es otra cosa que poner los medios suficientes para que los recursos humanos mejoren su competencia se realicen y desarrollen personalmente, alcanzando los objetivos, que no son otros que dar calidad al servicio sanitario, mejorando la salud de la población, y aumentando la satisfacción tanto del trabajador como del usuario.

El paciente, como el profesional de la salud, deben entender que la calidad de la atención clínica, viene precedida por la premisa de promocionar la salud de manera consciente, auto controlando sus pensamientos, sentimientos y acciones para evitar caer en los extremos, defectos y/o excesos que alteren su equilibrio dinámico o de salud, poniendo de paso a prueba este conocimiento de la salud para crear, cultivar y desarrollar la experiencia vivencial de lo real que abra el camino nada más y nada menos que para promulgar una cultura que le permita al usuario y a la comunidad toda una movilización social en procura de concientizarse sobre la importancia de deselitizar la salud para devolverle, en derecho, lo que siempre le ha pertenecido a esa comunidad, la dignificación del servicio.

El derecho a la salud se constituye en un reto para la humanidad, pero lo es aún más cuando con quien se está interactuando es con singularidades, por eso la salud es el resultado de los cuidados propios y hacia los demás, de la capacidad de tomar decisiones y controlar la vida propia, de asegurar de que los derechos del enfermo, deben ir a la par de la calidad, de la satisfacción de las necesidades del usuario y la satisfacción de qué tan bien educados e informados estén los pacientes y promotores de la salud a la hora de salvaguardar el bienestar y calidad de vida de la población, que no busca otra cosa que el de fomentar un alto nivel de excelencia del personal de salud, de un compromiso institucional de ese personal en el trato con el paciente y su familia con la seguridad de que todo aquel que llegue a un centro de salud, se lleve consigo una sensación agradable de querer volver.

Conflicto de interés: El autor declara no tener ningún conflicto de interés.

\section{Referencias}

1. Hamburger-Fernández AA. Humanización de la empresa. Colombia. Editorial Bonaventuriana, 2008: 4647.

2. Foucault M. El nacimiento de la clínica. México. Siglo XXI Editores. 1997:85-96.

3. Moscati P. De L'emplo; des systèmes dans la médicine pratique en Foucault, Michel. El nacimiento de la clínica. México Siglo XXI Editores. 1997:86.

4. Tissot, "Memoire por la construction d' un hopitaux clinique" en essai sur les études médicales (Lausana,1875) en Fuocault, Michel op cit, p 92.

5. Etchegoyen R. Horacio. Los fundamentos de la técnica psicoanalítica. España. Amorrortu Editores. 1984-85. p.19-20.

6. Fundación Acción Pro Derechos Humanos. Derechos Humanos. Net. Herramientas para la Defensa y Promoción de los Derechos Humanos. Disponible en: http://www.derechoshumanos.net/normativa/normas 
/1948-DeclaracionUniversal.htm? gclid=Cj0KEQjwkvrBRDwoMLav-219KIBEiQAUTkDU8nETBIqyHxumyO MrMUxpPIV0bbFEV8CtKdNn2aGdFIaAqhw8P8HAQ

7. Rodríguez CE. Enfoque de humanización de la atención desde la Acreditación. Director de Acreditación en Salud, ICONTEC. Revista Normas y Calidad (Salud y Bienestar). 2012;13:9-10.

8. Eliot T. S. La parte del todo. Poemas y juegos completos. New York. Harccurt Brace and World Inc. 1962:329

9. Buber M. Yo y tú. New Cork. Scribens and Sens. 1970:124.

10. Foucault M. Vigilar y Castigar. Buenos Aires: Siglo XXI Editores; 1976.

11. Herrera-Carranza M. Cómo instaurar un plan de calidad asistencial en un servicio clínico. España. Servicio de Cuidados Críticos y Urgencias. Hospital "Juan Ramón Jiménez" Huelva. Ediciones Ltda. 2001:42-43.

12. Donabedian A. La calidad de la atención médica. Ed. La Prensa Medica Mexicana. México, 1984.

13. Nino CS. Ética y derechos humanos. Buenos Aires. Edit. Paidós.1984: 20.

14. Pérez-Luño AE. Delimitación conceptual de los derechos humanos, en los derechos humanos. Estatuto Jurídico y sistemas. México. Océano 1983:39.

15. República de Colombia. Decálogo de los derechos de los pacientes. Resolución 13437 de 1991, por la cual se constituyen los Comités de Ética Hospitalaria y se adoptan el Decálogo de los derechos de los pacientes. Citado el 17 de junio de 2015. http://www.minsalud.gov.co/Normatividad/RESOLUC I\%C3\%93N\%2013437\%20DE\%201991.pdf

16. El Espectador. Redacción Salud. Continúa en aumento tutelas de salud en Colombia. http://www.elespectador.com/noticias/salud/continu a-aumento-numero-de-tutelas-de-salud-colombiaarticulo-553595

17. Papacchini A. Filosofía y derechos humanos. Cali Edit. Facultad de Humanidades. 1954:52.

18. Aranda P. Epidemiología general. ULA Consejo de Publicaciones. 1994. Tomo I:1. 